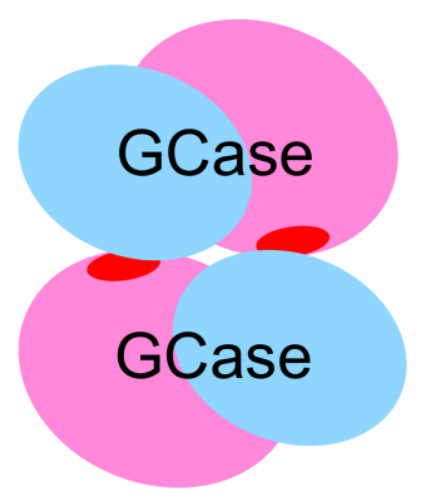

$+\underset{\rightleftarrows}{\stackrel{\text { Sap C }}{\rightleftarrows}}$

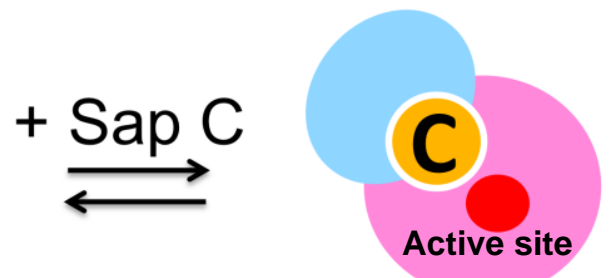

GCase

GCase

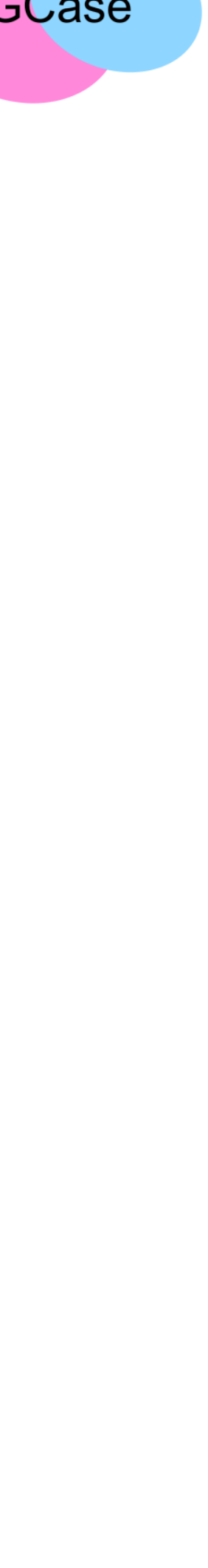




\title{
Dissociation of glucocerebrosidase dimer in solution by its co-factor, saposin $\mathrm{C}$
}

\author{
James M. Gruschus ${ }^{\mathrm{a}, *}$, Zhiping Jiang ${ }^{\mathrm{a}}$, Thai Leong Yap ${ }^{\mathrm{a}}$, Stephanie A. Hill ${ }^{\mathrm{a}}$, \\ Alexander Grishaev ${ }^{b}$, Grzegorz Piszczek ${ }^{c}$, Ellen Sidransky ${ }^{d}$, and Jennifer C. Lee ${ }^{\mathrm{a}, *}$
}

${ }^{a}$ Laboratory of Molecular Biophysics, Biochemistry and Biophysics Center, National Heart, Lung, and Blood Institute, National Institutes of Health, Bethesda MD 20892, United States

${ }^{b}$ Laboratory of Chemical Physics, National Institute of Diabetes and Digestive and Kidney Diseases, National Institutes of Health, Bethesda MD 20892, United States

${ }^{c}$ Biophysics Core, Biochemistry and Biophysics Center, National Heart, Lung, and Blood Institute, National Institutes of Health, Bethesda MD 20892, United States

${ }^{\mathrm{d}}$ Section on Molecular Neurogenetics, Medical Genetics Branch, National Human Genome Research Institute, National Institutes of Health, Bethesda MD 20892, United States

*corresponding authors.

J.M.G. E-mail: gruschuj@helix.nih.gov, Phone: 301-496-2350

J.C.L. E-mail: leej4@mail.nih.gov, Phone 301-496-3741 


\begin{abstract}
Mutations in the gene for the lysosomal enzyme glucocerebrosidase (GCase) cause Gaucher disease and are the most common risk factor for Parkinson disease (PD). Analytical ultracentrifugation of $8 \mu \mathrm{M}$ GCase shows equilibrium between monomer and dimer forms. However, in the presence of its co-factor saposin C (Sap C), only monomer GCase is seen. Isothermal calorimetry confirms that Sap C associates with GCase in solution in a 1:1 complex $\left(K_{\mathrm{d}}=2.1 \pm 1.1 \mu \mathrm{M}\right)$. Saturation cross-transfer NMR determined that the region of Sap C contacting GCase includes residues $63-66$ and 74-76, which is distinct from the region known to enhance GCase activity. Because $\alpha$-synuclein ( $\alpha$-syn), a protein closely associated with PD etiology, competes with Sap C for GCase binding, its interaction with GCase was also measured by ultracentrifugation and saturation cross-transfer. Unlike Sap C, binding of $\alpha$-syn to GCase does not affect multimerization. However, adding $\alpha$-syn reduces saturation cross-transfer from Sap C to GCase, confirming displacement. To explore where Sap C might disrupt multimeric GCase, GCase x-ray structures were analyzed using the program PISA, which predicted stable dimer and tetramer forms. For the most frequently predicted multimer interface, the GCase active sites are partially buried, suggesting that Sap C might disrupt the multimer by binding near the active site.
\end{abstract}

Keywords: Gaucher disease, Parkinson disease, NMR, AUC, $\alpha$-synuclein

Abbreviations: GCase, glucocerebrosidase; GluCer, glucosylceramide; GD, Gaucher disease; PD, Parkinson disease; $\alpha$-syn, $\alpha$-synuclein; Sap C, saposin C; AUC, analytical ultracentrifugation ITC, isothermal titration calorimetry; NMR, nuclear magnetic resonance; NaOAc, sodium acetate; PDB, Protein Data Bank. 


\section{Introduction}

GBA1 encodes glucocerebrosidase (GCase), a $60 \mathrm{kDa}$ lysosomal enzyme that hydrolyzes glucose from glucosyl-sphingolipids such as glucosylceramide (GluCer). Inheritance of mutated GBA1 from both parents results in the lysosomal storage disorder, Gaucher disease (GD). Mutations in $G B A 1$ are the most common known genetic risk modifiers for Parkinson disease (PD) [1]. Despite a wealth of studies linking GBA1 mutations and PD, the underlying mechanisms have yet to be defined [2,3]. Progress is being made, however, as a growing number of studies show a correlation between GCase deficiency and increased levels of $\alpha$ synuclein ( $\alpha$-syn), a protein closely associated with PD $[4,5,6]$. In fact, $\alpha$-syn physically interacts with GCase and inhibits its activity under the acidic conditions found in lysosomes [7,8]. Curiously, while GBA1 mutations are a common PD risk factor, the penetrance is low [9]. Only a minority of GD patients and carriers develop PD, thus other factors are also expected to play a role in promoting PD pathogenesis. Obvious molecules of interest include ones that modulate GCase activity and the interaction of $\alpha$-syn and GCase.

In vivo degradation of GluCer by GCase is facilitated by the co-factor saposin C (Sap C), a $9 \mathrm{kDa}$ membrane-interacting lysosomal protein [10]. The structures of GCase and Sap C are shown in Fig. 1. Sap C is one of four saposin proteins resulting from proteolytic cleavage of the $70 \mathrm{kDa}$ precursor prosaposin. All four saposins promote hydrolysis of glycolipids by various lysosomal enzymes $[11,12]$. Sap C has been proposed to aid GluCer hydrolysis by altering lipid bilayer properties, by enhancing recruitment of GCase to the membrane, or through a direct association with GCase that enhances its activity [13,14,15]. Although rare, Sap C deficiency alone can result in GD-like symptoms in patients [11], demonstrating its essential role in GluCer 
metabolism. We previously found that Sap C can fully rescue the inhibition of GCase activity by $\alpha$-syn, physically displacing the $\alpha$-syn bound to GCase [16].

For the fluorescence measurements of the displacement of $\alpha$-syn from GCase by Sap C, the results were best fit by a model where the proteins interact with a stoichiometry of 1:2 Sap C to GCase, implying either that Sap C has two sites that bind GCase monomers with comparable affinity or that one Sap C monomer binds to a dimer of GCase [16]. The same stoichiometry was obtained both for $\alpha$-syn displacement in solution and in the presence of lipid vesicles. Here, we report on a detailed characterization of the interaction of Sap C and GCase in solution. To test whether Sap C binds GCase dimer, we measure GCase using analytical ultracentrifugation (AUC) alone and in the presence of Sap C. Using isothermal titration calorimetry (ITC) we measure the stoichiometry and binding affinity. Finally, to test whether Sap C has two binding sites for GCase, we employ the saturation cross-transfer nuclear magnetic resonance (NMR) technique to map the interacting regions of Sap C with GCase in solution.

\section{Materials and Methods}

\subsection{Protein expression and purification}

The Sap C plasmid (pET-16, Novagen) was provided by Gilbert Privé (University of Toronto, Canada), and includes an additional Met-Gly at the N-terminus. Uniformly ${ }^{2} \mathrm{H} /{ }^{15} \mathrm{~N}$ labeled protein was produced by a procedure similar to that described in Deshmukh et al. [17] (additional details provided in Fig. S2). Mass spectrometric analysis indicated the loss of the Nterminal methionine and $97 \%$ deuteration of non-exchangeable sites $\left(9,588 \mathrm{Da}\right.$ in $\left.\mathrm{H}_{2} \mathrm{O}\right) .{ }^{2} \mathrm{H} /{ }^{15} \mathrm{~N}$ 
human recombinant $\alpha$-syn was expressed similarly but in BL21(DE3) pLysS cells. $\alpha$-Syn was purified as previously described [18] and mass spectrometry indicated 99\% deuteration of nonexchangeable sites $\left(15,405 \mathrm{Da}\right.$ in $\left.\mathrm{H}_{2} \mathrm{O}\right)$. Taliglucerase alfa (recombinant GCase) was obtained as a gift from Protalix Biotherapeutics Corp. (Carmiel, Israel). Except where noted, all samples were exchanged into $50 \mathrm{mM}$ sodium acetate $(\mathrm{NaOAc})$ and $100 \mathrm{mM} \mathrm{NaCl}$ buffer (pH 5) by repeated buffer exchanges using Amicon Ultra YM3 (Sap C and $\alpha$-syn) and YM30 (GCase) centrifugal filters (Millipore). Protein concentrations were determined using theoretical extinction coefficients at $280 \mathrm{~nm}: \varepsilon_{280}($ GCase $)=94,353 \mathrm{M}^{-1} \mathrm{~cm}^{-1}, \varepsilon_{280}($ Sap C $)=3,355 \mathrm{M}^{-1}$ $\mathrm{cm}^{-1}$, and $\varepsilon_{280}(\alpha-\mathrm{syn})=5,120 \mathrm{M}^{-1} \mathrm{~cm}^{-1}$.

\subsection{Analytical ultracentrifugation (AUC)}

All AUC experiments were performed at $20^{\circ} \mathrm{C}$ in the ProteomeLab XL-I analytical ultracentrifuge (Beckman Coulter, Indianapolis, IN) using absorption optics as previously described [19]. Samples with GCase and $2 \mu \mathrm{M}$ Sap C ( $\alpha$-syn) were prepared and measured first and then the $20 \mu \mathrm{M}$ samples were made by adding appropriate volumes of higher concentration stocks of Sap C ( $\alpha$-syn) and concentrating to the original sample volume. Final sample concentrations were verified by absorbance at $280 \mathrm{~nm}$; a lower GCase concentration $(\sim 5$ vs. 7 $\mu \mathrm{M})$ was measured for the sample with $20 \mu \mathrm{M} \alpha$-syn. Data were analyzed in terms of continuous distributions $(c(s))$ using the SEDFIT program $[19,20]$ with RMSD $<0.004$ and were plotted in GUSSI (http://biophysics.swmed.edu/MBR/software.html).

\subsection{Isothermal titration calorimetry (ITC)}

ITC titrations were performed at $25^{\circ} \mathrm{C}$ using the ITC200 calorimeter (MicroCal, Northampton, MA). GCase $(20 \mu \mathrm{M})$ in the $200 \mu \mathrm{L}$ cell was titrated with $300 \mu \mathrm{M}$ Sap C using 30 
injections at 300-s intervals. The first injection was $0.2 \mu \mathrm{L}$ and all others were $1.3 \mu \mathrm{L}$ up to 3 molar equivalents. Data were integrated using NITPIC [21], were analyzed in SEDPHAT [22], and plotted in GUSSI. Syringe concentration (Sap C incompetent fraction) and heat of dilution were adjusted during the fit.

\subsection{Nuclear magnetic resonance (NMR)}

The saturation cross-transfer experiment was performed as described [23] and consists of a WURST adiabatic pulse repeated for $3.2 \mathrm{~s}$ over the frequency range desired for saturation, followed by a standard ${ }^{15} \mathrm{~N}$ HSQC pulse sequence. The pulse sequence was also repeated for each time point with the saturation frequency range far off resonance, at roughly $-1667 \mathrm{ppm}$ in the proton dimension, and collected interleaved with the on-resonance data. The off-resonance spectra backbone amide intensities (peak heights) were used to normalize the on-resonance intensities. The normalized intensities in the presence of GCase were corrected for solvent effects by dividing by the normalized intensities of Sap C alone. The NMR experiments were performed at $15^{\circ} \mathrm{C}$ on a Bruker $600 \mathrm{MHz}$ Avance spectrometer equipped with a cryoprobe, with $5 \% \mathrm{D}_{2} \mathrm{O}$ added to the $50 \mathrm{mM} \mathrm{NaOAc}, 100 \mathrm{mM} \mathrm{NaCl} \mathrm{pH} 5$ buffer. Sap C assignments are from de Alba et al. [24].

\section{Results}

\subsection{Sap C binds to GCase monomer}

Preliminary work on GCase using small-angle X-ray scattering at concentrations $\geq 22$

$\mu \mathrm{M}$ in solution indicated GCase was present in multiple multimeric forms (Fig. S1). To explore 
multimerization of GCase at lower concentrations and to probe the impact of Sap C interaction, AUC was empoyed. GCase has 12 tryptophan residues, allowing measurements using UV absorbance at $280 \mathrm{~nm}$ down to $\sim 1 \mu \mathrm{M}$. The $c\left(s_{20, w}\right)$ distribution of GCase at $8.0 \mu \mathrm{M}$ shows two main peaks consistent with monomer $(4.4 S)$ and dimer (5.9 $S$ ) (see below), with trace amounts of higher molecular weight species and aggregates $(<4 \%$ total concentration) (Fig. 2A). The populations of the putative monomer and dimer are nearly equivalent $(53 \%$ and $47 \%$ for monomer and dimer species, respectively) suggesting that the $K_{\mathrm{d}}$ for GCase dimerization is in the $\mu \mathrm{M}$ range. At $1.7 \mu \mathrm{M}$, only the monomer peak is present with a slightly lower sedimentation coefficient (4.2 $S$ ) and with a broad shoulder extending to the higher $S$ values (Fig. 2A), indicating a fast dimerization off-rate on the AUC experiment time-scale $\left(k_{\text {off }}>10^{-2} \mathrm{~s}^{-1}\right)$. For 7.4 $\mu \mathrm{M}$ GCase in the presence of $2 \mu \mathrm{M}$ Sap $\mathrm{C}$, the pattern of oligomerization is unchanged and there is no appearance of a signal for free Sap C, suggesting that all Sap C is bound (Fig. 2B). Surprisingly, when Sap C is increased to $20 \mu \mathrm{M}$, only the monomeric GCase peak is observed at 4.4 $S$ (Fig. 2B), slightly higher than the $1.7 \mu \mathrm{M}$ monomeric peak $(4.2 S)$, thus Sap $\mathrm{C}$ is bound to and keeps GCase in its monomeric state. Note that the relative positions of the Sap C, GCase monomer and dimer peaks 1.2 S, 4.2 S, and 5.9 S are consistent with simple spherical model predictions using their molecular weight ratios to the $2 / 3$ power, i.e. $(60 \mathrm{kD} / 9 \mathrm{kD})^{2 / 3} \sim(4.2 S / 1.2 S)$ and $(120 \mathrm{kD} / 60 \mathrm{kD})^{2 / 3} \sim(5.9 S / 4.2 S)$.

Sap C and $\alpha$-syn compete for GCase binding [16], so $\alpha$-syn was tested to see if it also could affect dimerization. As with Sap C, when 7.2 $\mu \mathrm{M}$ GCase was evaluated in the presence of $2 \mu \mathrm{M} \alpha$-syn, the pattern of oligomerization was not changed, and the absence of a signal for free $\alpha$-syn indicated that all $\alpha$-syn is bound (Fig. 2C). Unlike with Sap C, increasing the $\alpha$-syn concentration to $20 \mu \mathrm{M}$ did not prevent dimerization of GCase, even with a lower GCase 
concentration $(5.0 \mu \mathrm{M})($ Fig. 2C). Instead, at the higher concentration $\alpha$-syn causes both GCase peaks to broaden and shift towards smaller $S_{20, \mathrm{w}}$ values, suggesting that $\alpha$-syn might be interacting with both monomer and dimer forms of GCase. Note that when $\alpha$-syn binds to GCase in solution, residues $1-117$ of $\alpha$-syn do not contact GCase and remain intrinsically disordered [7]. The $S_{20, w}$ value of the complex depends not only on the total mass, but on the specific density and shape factor as well, so the non-interacting flexible region of $\alpha$-syn might account for the changes in the sedimentation peaks.

\subsection{Sap C binds GCase with 1:1 stoichiometry}

The raw and baseline corrected thermograms, along with the fit to the integrated peaks with residuals, are shown in Fig. 3. The titration of Sap C into GCase resulted in the exothermic reaction with the apparent enthalpy of $-4.1 \mathrm{kcal} / \mathrm{mol}$. The binding isotherm was fit using models assuming 1:1, 2:1 or 1:2 Sap C/GCase association ratios. The 1:1 model yielded the best fit with an incompetent fraction of $18 \%$ for Sap C. The other association ratios yielded unrealistically high incompetent fraction values, $40 \%$ incompetent GCase for the 1:2 model and $60 \%$ incompetent Sap C for the 2:1 model. The 1:1 model yielded a $K_{\mathrm{d}}$ of $2.1 \pm 1.1 \mu \mathrm{M}$ with a heat of dilution of $-0.75 \mathrm{kcal} / \mathrm{mol}$ for Sap C.

\subsection{Region of Sap C in contact with GCase}

In order to determine which Sap C residues are in contact with GCase, a saturation crosstransfer NMR experiment was performed [23]. In this experiment, the GCase methyl group ${ }^{1} \mathrm{H}$ spin populations are first saturated, then diffuse via NOE interactions across the interface to the Sap C backbone amide protons closest to the GCase. The Sap C in the sample must be deuterated, $97 \%$ in our case determined by mass spectrometry, in order to minimize direct 
saturation of the ${ }^{1} \mathrm{H}$ population in Sap C. In order to gauge the impact of residual methyl protonation of Sap C and other potential effects, such as interaction with solvent, the saturation cross-transfer experiment was first performed on $38 \mu \mathrm{M}$ Sap C alone. The results for the backbone amides for Sap $\mathrm{C}$ alone are shown in Fig. 4A, and the corresponding $2 \mathrm{D}^{15} \mathrm{~N}$ HSQC spectrum in shown in Fig. S2A. The experiment was performed twice using two saturation sweep widths (0.1-1.1 and $0.05-0.55 \mathrm{ppm})$, indicated by the error bars, and no qualitative difference was observed between the data sets. Both data sets show intensity reduction for residues $45-47$ at the beginning of the third helix of Sap C. Since the backbone amides of these residues are not near any methyl groups it is unclear what causes this reduction, perhaps some effect involving the solvent.

The results for the saturation cross-transfer experiment in the presence of $33 \mu \mathrm{M}$ GCase are shown in Fig. 4B, with the corresponding ${ }^{15} \mathrm{~N}$ HSQC spectrum shown in Fig. S2A. GCase causes the greatest reduction for Sap C residues 63-66, which lie in the loop preceding the final helix. Residues 74-76 at the end of the final helix also show significant reduction. The location of these residues in the human and consensus Sap C sequences are shown in Fig. S3. The experiment was repeated with GCase at $11 \mu \mathrm{M}$ (Fig. 4B), and less intensity reduction for residues $63-66$ and 74-76 was observed, confirming that their signal reduction comes from saturation cross-transfer. Sap C and $\alpha$-syn compete for GCase binding, and this was confirmed using saturation cross-transfer, where added $\alpha$-syn $(152 \mu \mathrm{M})$ also caused less intensity reduction in residues 63-66 and 74-76 of Sap C (Fig. 4C). As an additional control, saturation crosstransfer between GCase and ${ }^{2} \mathrm{H} /{ }^{15} \mathrm{~N} \alpha$-syn was measured at two GCase concentrations with residues 122-137 showing the greatest intensity reduction (Fig. S4). This corresponds well to 
results using backbone amide intensity reduction in ${ }^{15} \mathrm{~N}$ HSQC NMR spectra [7], where $\alpha$-syn residues 118-137 were determined as the region of interaction.

\section{Discussion}

The AUC results clearly show that GCase exists in monomer/multimer equilibrium in solution that is driven to the monomeric state when interacting with Sap C. ITC confirmed a 1:1 complex, though it is unclear whether Sap C actively dissociates GCase dimer or simply binds free monomer and prevents self-association. The 1:1 stoichiometry contradicts earlier proposals [25], including our own based on displacement of $\alpha$-syn by Sap C probed by fluorescence spectroscopy, where the data was best fit with a 1:2 Sap C/GCase binding model [16]. The model assumed non-cooperative, independent binding sites. However, since Sap C binding involves transition of GCase from multimer to monomer states (Fig. 2B), the assumption of noncooperative binding is likely not valid, potentially accounting for the discrepancy.

The concentration of $8 \mu \mathrm{M}$ used in the AUC experiments might seem high for the cellular milieu; however, GCase is trafficked to lysosomes confined inside vesicles. A simple calculation ( $n=(\pi / 6) c d^{3} N_{\mathrm{A}}$, where $n$ is the number of molecules, $c$ is the concentration in molar, $d$ is the diameter in decimeters, and $N_{\mathrm{A}}$ is Avogadro's number) shows that just 19 molecules of GCase in a vesicle with inner diameter $200 \mathrm{~nm}$ yields a concentration of $8 \mu \mathrm{M}$. To give a sense of the dimensions involved, GCase measures $9.5 \mathrm{~nm}$ along its widest axis, so the vesicle is over 20 times wider. In a study of GCase interacting with its trafficking partner LIMP-2, preliminary cross-linking results suggested they form a 2:2 complex [26], and while this does not prove GCase binds LIMP-2 as a dimer, it is consistent with the possibility. 
While the LIMP-2 cross-linking results hint that dimeric GCase might be present in trafficking vesicles, there is also evidence of the importance of monomeric GCase in vivo from studies using radiation inactivation of human spleen homogenates [27]. This experiment measures the homo-multimerization state of enzymes. For homogenates from normal spleens, GCase was found to be predominantly monomeric. Intriguingly, in homogenates from spleens of two GD patients, GCase was predominantly dimeric. This suggests the mutations in these particular GD patients cause GCase to favor a dimeric form. Building on this idea, one of the biological roles of Sap C could be to facilitate the transition of GCase to a monomeric form in the lysosome; hence, there could be mutations that promote GD and PD because they interfere with this aspect of the Sap C interaction.

The NMR results presented here map the region contacted by GCase to Sap C residues 63-66 and 74-76. Two previous experimental studies have probed which part of Sap C interacts with GCase and enhances its activity $[25,28]$. Both studies found that the second half of Sap C activates GCase nearly as well as intact Sap C. Interestingly, both studies also found that the residues most important for activation lie in the region just before that identified by NMR, with the first study identifying residues 41-49 [25] and the second residues 47-62 [28]. These results are consistent with proposed mechanisms where Sap C alters membrane structure in a manner that facilitates substrate access to GCase and helps to recruit GCase to the altered membrane region $[14,29]$. We note that in the first study, the most strongly binding peptide included residues $41-80$, with an apparent dissociation constant ( $K_{\text {act }}$ in their nomenclature) of $1.3 \mu \mathrm{M}$, similar to the $K_{\mathrm{d}}$ reported here.

The precise region of GCase that Sap C binds to has not been determined experimentally. The disruption of dimeric GCase by Sap C suggests that one possibility is that the Sap C binding 
site overlaps with the dimer interface. In order to learn more about which multimer forms GCase might assume, we surveyed the x-ray structures of human GCase using the PDBe PISA program for predicting energetically favorable macromolecular interfaces [30]. Nearly all the structures were predicted to form stable dimers and/or tetramers, with the lowest free energy, most frequently predicted multimer interface having the active sites facing the interface and partially buried (Fig. S5). Thus, if the Sap C binding site overlaps with the dimer interface, then the PISA results would suggest that Sap C binds somewhere near the enzyme active site.

\section{Acknowledgement}

This work was supported by the Intramural Research Program at the National Institutes of Health, National Heart, Lung, and Blood Institute (NHLBI), National Institute of Diabetes and Digestive and Kidney Diseases (NIDDK), and the National Human Genome Research Institute (NHGRI). Recombinant GCase was a gift from Protalix Biotherapeutics (Carmiel, Israel). The Sap C plasmid was provided by Gilbert Privé (University of Toronto, Toronto, ON). We thank Nico Tjandra (NHLBI) for his advice regarding the NMR experiments and the use of NMR spectrometers, and Duck-Yeon Lee (NHLBI Biochemistry Core Facility) for his assistance with the mass spectrometry and analysis. We thank S. Seifert and X. Zuo for assistance with SAXS data collection and gratefully acknowledge use of the Advanced Photon Source, supported by the U.S. Department of Energy, contract No. W-31-109-ENG-38, and the shared scattering beamline resource allocated under the PUP-77 agreement between NCI, NIH, and the Argonne National Laboratory. 


\section{REFERENCES}

[1] E. Sidransky, M.A. Nalls, J.O. Aasly, et al., Multicenter analysis of glucocerebrosidase mutations in Parkinson's disease, N. Engl. J. Med. 361 (2009) 1651-1661.

[2] W. Westbroek, A.M. Gustafson, E. Sidransky, Exploring the link between glucocerebrosidase mutations and parkinsonism, Trends Mol. Med. 17 (2011) 485-493.

[3] R.P. McGlinchey, J.C. Lee, Emerging insights into the mechanistic link between $\alpha$-synuclein and glucocerebrosidase in Parkinson's disease, Biochem Soc. Trans. 41 (2013) 15091512.

[4] V. Cullen, S.P. Sardi, J. Ng, et al., Acid $\beta$-glucosidase mutants linked to Gaucher disease, Parkinson disease, and Lewy body dementia alter $\alpha$-synuclein processing, Ann. Neurol. 69 (2011) 940-953.

[5] J.R. Mazzulli, Y.H. Xu, Y. Sun, et al., Gaucher disease glucocerebrosidase and $\alpha$-synuclein form a bidirectional pathogenic loop in synucleinopathies, Cell 146 (2011) 37-52.

[6] S.P. Sardi, J. Clarke, C. Kinnecom, et al., CNS expression of glucocerebrosidase corrects $\alpha-$ synuclein pathology and memory in a mouse model of Gaucher-related synucleinopathy, Proc. Natl. Acad. Sci. U. S. A. 108 (2011) 12101-12106.

[7] T.L. Yap, J.M. Gruschus, A. Velayati, et al., $\alpha$-Synuclein interacts with Glucocerebrosidase providing a molecular link between Parkinson and Gaucher diseases, J. Biol. Chem. 286 (2011) 28080-28088.

[8] T.L. Yap, A. Velayati, E. Sidransky, et al., Membrane-bound $\alpha$-synuclein interacts with glucocerebrosidase and inhibits enzyme activity, Mol. Genet. Metab. 108 (2013) 56-64.

[9] E. Sidransky, P.S. Hart, Penetrance of PD in glucocerebrosidase gene mutation carriers, Neurology 79 (2012) 106-107.

[10] T. Kolter, K. Sandhoff, Principles of lysosomal membrane digestion: stimulation of sphingolipid degradation by sphingolipid activator proteins and anionic lysosomal lipids, Annu. Rev. Cell Dev. Biol. 21 (2005) 81-103.

[11] R.J. Tamargo, A. Velayati, E. Goldin, et al., The role of saposin C in Gaucher disease, Mol. Genet. Metab. 106 (2012) 257-263.

[12] J. Matsuda, M. Kido, K. Tadano-Aritomi, et al., Mutation in saposin D domain of sphingolipid activator protein gene causes urinary system defects and cerebellar Purkinje cell degeneration with accumulation of hydroxy fatty acid-containing ceramide in mouse, Hum. Mol. Genet. 13 (2004) 2709-2723.

[13] J.R. Alattia, J.E. Shaw, C.M. Yip, et al., Molecular imaging of membrane interfaces reveals mode of $\beta$-glucosidase activation by saposin C, Proc. Natl. Acad. Sci. U.S.A. 104 (2007) 17394-17399.

[14] A.M. Vaccaro, M. Tatti, F. Ciaffoni, et al., Effect of saposins A and C on the enzymatic hydrolysis of liposomal glucosylceramide, J. Biol. Chem. 272 (1997) 16862-16867.

[15] X.Y. Qi, G.A. Grabowski, Acid $\beta$-glucosidase: Intrinsic fluorescence and conformational changes induced by phospholipids and saposin C, Biochemistry 37 (1998) 11544-11554.

[16] T.L. Yap, J.M. Gruschus, A. Velayati, et al., Saposin C protects glucocerebrosidase against a-synuclein inhibition, Biochemistry 52 (2013) 7161-7163.

[17] L. Deshmukh, C.D. Schwieters, A. Grishaev, et al., Structure and dynamics of full-length HIV-1 capsid protein in solution, J. Am. Chem. Soc. 135 (2013) 16133-16147.

[18] C.M. Pfefferkorn, J.C. Lee, Tryptophan probes at the $\alpha$-synuclein and membrane interface, J. Phys. Chem. B 114 (2010) 4615-4622. 
[19] H. Zhao, R. Ghirlando, G. Piszczek, et al., Recorded scan times can limit the accuracy of sedimentation coefficients in analytical ultracentrifugation, Anal. Biochem. 437 (2013) 104-108.

[20] P. Schuck, Size-distribution analysis of macromolecules by sedimentation velocity ultracentrifugation and LAMM equation modeling, Biophys. J. 78 (2000) 1606-1619.

[21] S. Keller, C. Vargas, H. Zhao, et al., High-precision isothermal titration calorimetry with automated peak-shape analysis, Anal. Chem. 84 (2012) 5066-5073.

[22] P. Schuck, On the analysis of protein self-association by sedimentation velocity analytical ultracentrifugation, Anal. Biochem. 320 (2003) 104-124.

[23] T. Nakanishi, M. Miyazawa, M. Sakakura, et al., Determination of the interface of a large protein complex by transferred cross-saturation measurements, J. Mol. Biol. 318 (2002) 245-249.

[24] E. de Alba, S. Weiler, N. Tjandra, Solution structure of human saposin C: pH-dependent interaction with phospholipid vesicles, Biochemistry 42 (2003) 14729-14740.

[25] S. Weiler, Y. Kishimoto, J.S. O'Brien, et al., Identification of the binding and activating sites of the sphingolipid activator protein, saposin C, with glucocerebrosidase, Protein Sci. 4 (1995) 756-764.

[26] J. Blanz, J. Groth, C. Zachos, et al., Disease-causing mutations within the lysosomal integral membrane protein type 2 (LIMP-2) reveal the nature of binding to its ligand $\beta$ glucocerebrosidase, Hum Mol Genet 19 (2010) 563-572.

[27] A. Maret, M. Potier, R. Salvayre, et al., In vitro detergent activation of lysosomal acid $\beta$ glucosidase in the spleen of normal and type-1 Gaucher patients is not accompanied by change in aggregation state, Biochim. Biophys. Acta 996 (1989) 254-256.

[28] X. Qi, W. Qin, Y. Sun, et al., Functional organization of saposin C. Definition of the neurotrophic and acid $\beta$-glucosidase activation regions, J. Biol. Chem. 271 (1996) 68746880.

[29] J.R. Alattia, J.E. Shaw, C.M. Yip, et al., Direct visualization of saposin remodelling of lipid bilayers, J. Mol. Biol. 362 (2006) 943-953.

[30] E. Krissinel, K. Henrick, Inference of macromolecular assemblies from crystalline state, J. Mol. Biol. 372 (2007) 774-797. 


\section{FIGURE LEGENDS}

Figure 1. Structures of GCase and Sap C. (A) The ribbon structure of GCase is shown with the $\mathrm{N}$ - and $\mathrm{C}$ - terminal $\beta$-domains in light blue, the TIM barrel catalytic domain in pink with the catalytic glutamate residues in red, the common GD mutation site N370S in green, and glycosylation sites in yellow. (B) The ribbon structure of Sap C is shown shaded from red to violet from the $\mathrm{N}$ - to the $\mathrm{C}$-terminus. Locations of $\mathrm{N}$ - and $\mathrm{C}$-termini are also indicated.

Figure 2. AUC results for GCase in the presence of Sap C and $\alpha$-syn. (A) Sedimentation coefficients for GCase alone at $8.0 \mu \mathrm{M}$ (blue curve) and $1.7 \mu \mathrm{M}$ (purple curve). The sedimentation coefficient distributions $c(\mathrm{~s})$ are plotted in relative absorbance units versus svedbergs $(S)$. The monomer and dimer peaks are indicated by $\mathrm{M}$ and $\mathrm{D}$, respectively. (B) Sedimentation coefficients of 7.4 $\mu \mathrm{M}$ GCase in the presence of $2.0 \mu \mathrm{M}$ Sap C (blue), and 7.8 $\mu \mathrm{M}$ GCase in the presence of $20 \mu \mathrm{M}$ Sap $\mathrm{C}$ (purple). For $20 \mu \mathrm{M}$ Sap $\mathrm{C}$, the peak corresponding to GCase dimer is absent. The new peak seen at $S=1.2$ corresponds to unbound Sap C. (C) Sedimentation coefficients of 7.2 $\mu \mathrm{M}$ GCase in the presence of $2.0 \mu \mathrm{M} \alpha$-syn (blue), and $5.0 \mu \mathrm{M}$ GCase in the presence of $20 \mu \mathrm{M} \alpha$-syn (purple). The new peak seen at $S=1.2$ corresponds to unbound $\alpha$-syn.

Figure 3. ITC titration of GCase with Sap C. (A) Baseline corrected DP (measured power differential) peaks used for enthalpy determination. Inset shows the raw ITC data. Portions not included in the baseline corrected peaks are shown in black. (B) Plot of the integrated peaks with error bars determined by baseline uncertainty [21], fit with a 1:1 binding curve and (C) residuals resulting from the fit, ranging from -0.27 to $0.24 \mathrm{kcal} / \mathrm{mol}$. 
Figure 4. Saturation cross-transfer from GCase to Sap C. (A) Saturation cross-transfer backbone amide resonance intensities $\left(\mathrm{I}_{\mathrm{Sat}}\right)$ of $38 \mu \mathrm{M}$ Sap C alone, normalized by the intensities with the saturation applied off-resonance $\left(\mathrm{I}_{\mathrm{OR}}\right)$. The error bars indicate values from two experiments, with the saturation done at two different sweep widths. (B) Sap C (38 $\mu \mathrm{M})$ saturation cross-transfer backbone amide resonance intensities in the presence of GCase at $33 \mu \mathrm{M}$ (dark blue) and $11 \mu \mathrm{M}$ (light blue), normalized by the off-resonance intensities, and corrected by the normalized intensities of Sap C alone (see Materials and methods), indicated by the primes ( $\left.\mathrm{I}_{\mathrm{Sat}}^{\prime} \mathrm{I}_{\mathrm{OR}}^{\prime}\right)$. The error bars indicate values from two experiments for GCase at $33 \mu \mathrm{M}$, with the saturation done at two different sweep widths. (C) Sap C $(38 \mu \mathrm{M})$ normalized, corrected saturation cross-transfer intensities ( $\mathrm{I}_{\mathrm{Sat}}^{\prime} \mathrm{I}_{\mathrm{OR}}^{\prime}$ ) with $33 \mu \mathrm{M}$ GCase in the presence (green) and absence (dark blue) of 152 $\mu \mathrm{M} \alpha$-syn. The dark blue curve is the same as in panel B and is shown for comparison. 

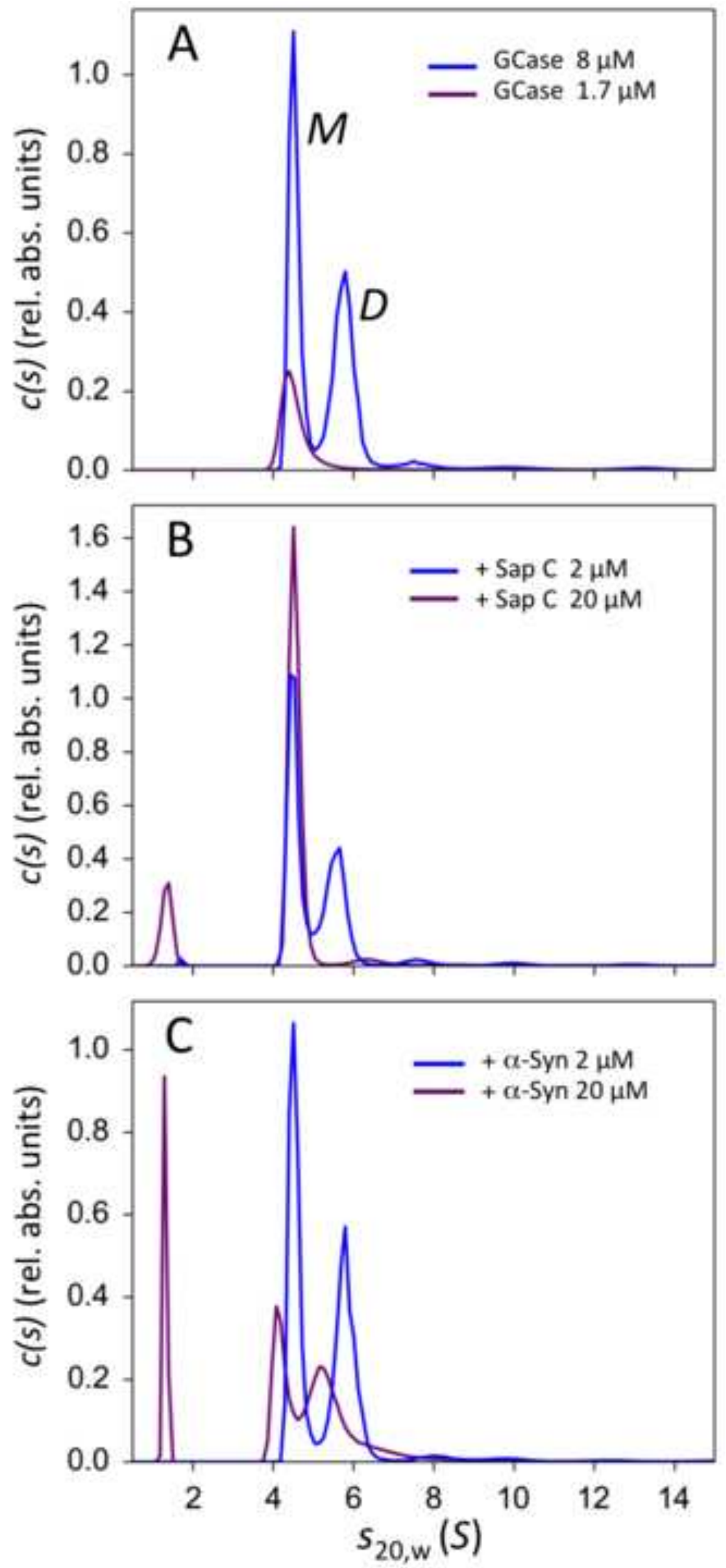


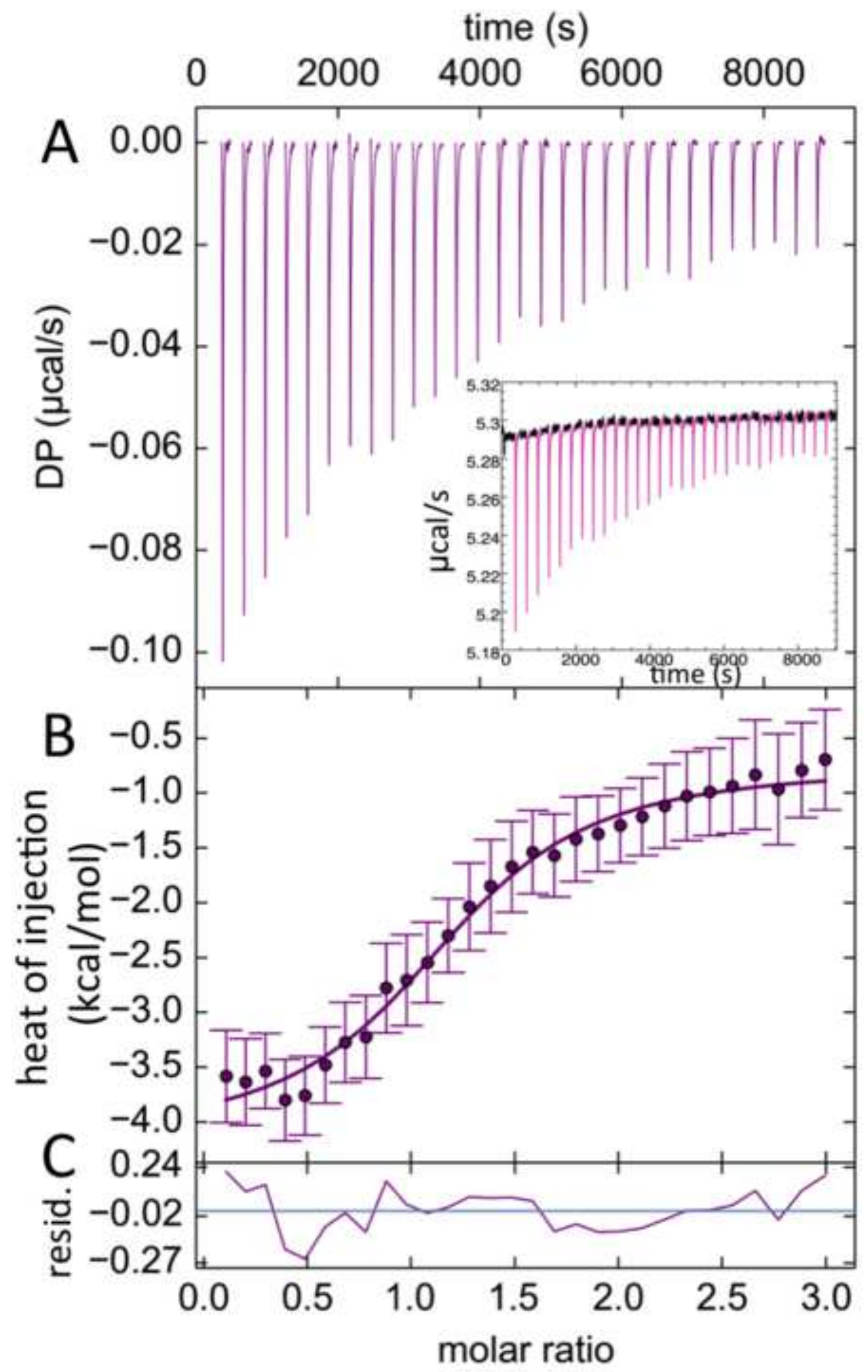



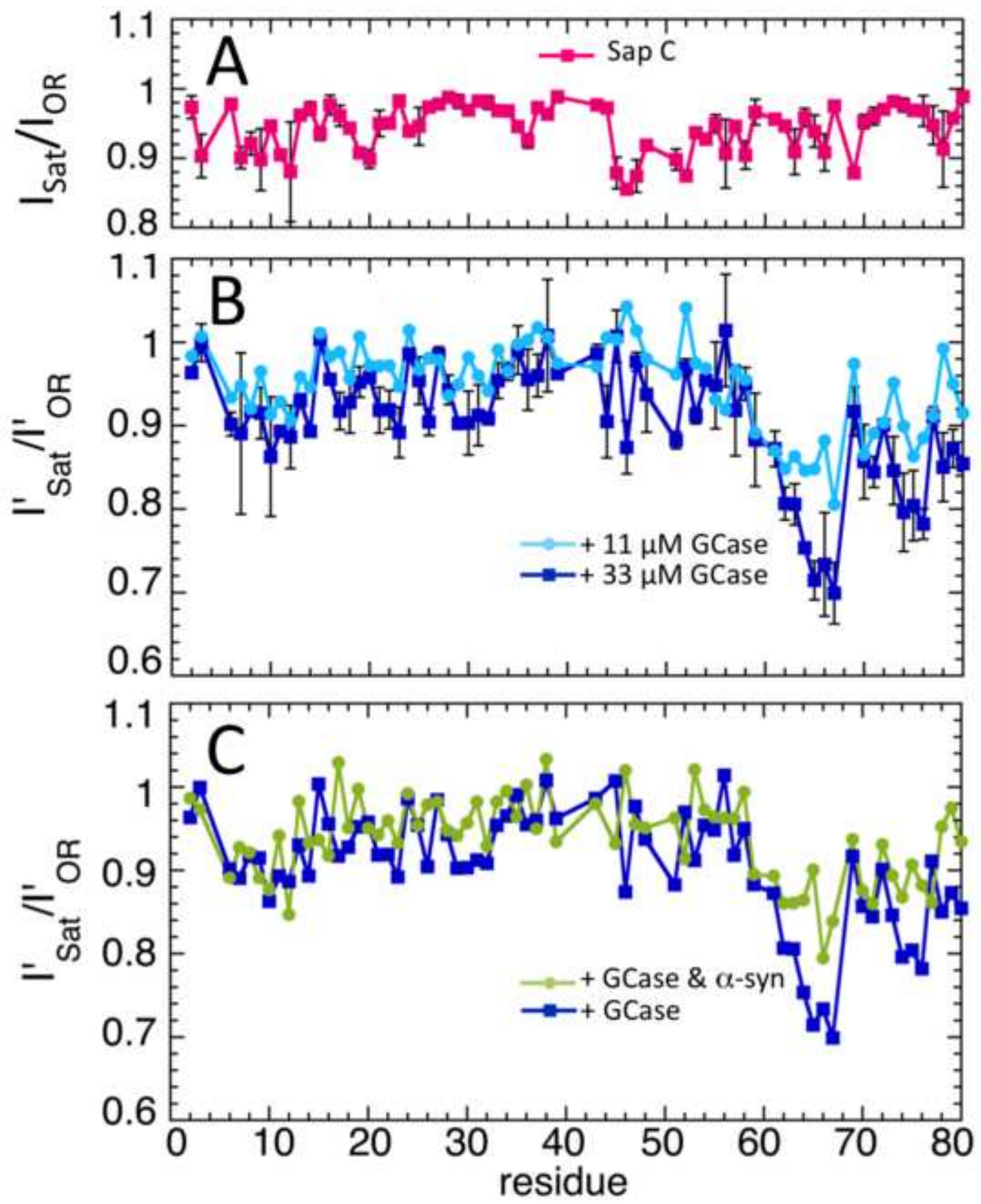